\title{
Effects of 8 Weeks of Plyometric Exercise on Certain Physiological Parameters and Performance of Swimmers
}

\author{
Yildirim Gokhan Gencer ${ }^{1}$, Emre Can Igdir ${ }^{1}$, Serdar Sahin ${ }^{1}$, Fatih Eris ${ }^{1}$ \\ ${ }^{1}$ Yuzuncu Yil University, School of Physical Education and Sports, Van, Turkey \\ Correspondence: Yildirim Gokhan Gencer, Yuzuncu Yil University, School of Physical Education and Sports, Van, \\ Turkey.
}

Received: April 9, 2018

Accepted: May 8, $2018 \quad$ Online Published: May 15, 2018

doi:10.11114/jets.v6i7.3194

URL: https://doi.org/10.11114/jets.v6i7.3194

\begin{abstract}
In this study, it is aimed to review the effects of 8 week plyometric exercises on certain physiological parameters and freestyle swimming performance of swimmers of age 10-11 who practice swimming with a licence in city of Van. Study group has been created by 24 male volunteer swimmers who practice swimming with a licence in city of Van. 24 volunteer athletes aged 10-11, who are licenced swimmers for two years and who exercise regularly 5 days a week have been selected for the study. The athletes are randomly divided into experimental and control groups of 12 . Throughout the study, in addition to swimming exercises, experimental group is scheduled to 3 days a week plyometric exercise program. Control group is not given any additional exercise except from the swimming exercises. It is seen that the obtained data does not show normal distribution and non-parametric tests of Mann Whitney $U$ and Wilcoxon Test are used for data analysis. Significance level is taken as $\mathrm{p}<0,05$. As per obtained data, pretest and post test data of male swimmers of experimental group show that horizontal and vertical leaps, anaerobic power, $30 \mathrm{~m}$ sprint, hand grip right and left, 25 and $50 \mathrm{~m}$ freestyle swimming parameters showed statistical significant difference; whereas for control group, weight, height, body mass index, anaerobic power and right and left hand grip force parameters showed statistical significant difference $(\mathrm{p}<0,05)$. When the pretest and posttests of experimental and control groups are compared, only vertical leap parameter showed significant difference in favor of the experimental group $(\mathrm{p}<0,05)$ and for the other parameters, no statistical significant difference is seen $(p>0,05)$. As a result, it is seen that the 8 week plyometric exercise has a significant effect on vertical leap and even though not statistically significant, it is pondered that it has a positive effect on freestyle swimming duration of experimental group, compared to that of control group.
\end{abstract}

Keywords: plyometric exercise, swimming, physiological parameter, performance

\section{Introduction}

When the human race settled down to pursue their existence in prehistoric times, they preferred to settle down by water. Among the reasons of this choice, there were the facts that food resources are richer by water and it is easier to be protected by wild animals there. Human beings then began swimming with more primitive techniques than that of today's modern swimming, in order to satisfy their hunger and to protect themselves from wild animals (Morpa, 2003; Bozdogan and Ozuak 2003). Since then, swimming has evolved and improved to become what it is today and is now divided into 4 different styles in international competitions.

Fastest one in these different swimming styles is the free style swimming. It consists of one right arm traction followed by the left and foot stomps (Bozdogan, 2003). Swimming as a sport is a branch that affects especially the upper extremity power. Attributes sought after in a successful swimmer are being tall, a large muscle mass, long upper and lower extremities and a wide shoulder structure (Troup, 1999). The sport of swimming is a complex sport branch which includes many factors such as high level anaerobic and aerobic capacity, stamina, force, a flexible body, velocity, speed, coordination and a good sense of rhythm (Tuzen et al., 2005). In swimming, the facts that lower extremity is powerful at a maximal level and its explosiveness are among the important variables which affect the performance of the athlete (Yapici et al., 2015).

There are various power and stamina exercises the trainers frequently turn to in order to increase the performance of the athletes as per their physical properties. For the athletes of pre-adolescence period, station exercises, performed with own body weight and coupled exercises are used as power developing methods (Sevim, 1991). These exercises can be 
performed both inside and outside water. Within the power exercises done on the ground, as per the age and physical properties of the athletes, jumping exercises can be done in addition to various other ones such as exercises performed with own body weight, exercises with auxiliary tools (bosu ball, thera band etc.) or exercises with small-medium or high weights (Yapici et al., 2016). Plyometric exercises, as it is a type of exercise which helps work every muscle of an athlete, is used as an effective method in increasing the power and speed capacity of athletes (Donald et al., 2003). This study aims to reveal the effect of 8 week of plyometric exercise method on certain physiological parameters and performance of swimmers aged 10-11.

\section{Method}

24 male athletes aged 10-11 from the city of Van who have a background of swimming with a license for two years voluntarily participated in the study, with consent forms obtained from their parents. 12 athletes are selected to undergo Pylometric exercises as experimental group and 12 athletes are selected to the control group. Random selection is used to set the groups. Experimental group is scheduled to perform Plyometric exercises 3 days a week in addition to their regular swimming exercises. Control group is requested to continue their regular daily swimming exercises. Selected measurements are obtained from the athletes before applying the exercise program and also after 8 weeks and then the change between pre and posttest values are reviewed.

Plyometric Exercise Program: Exercises are performed 3 days a week (Mondays, Wednesdays and Fridays). Resting periods are paid attention to (Nobre et al., 2017).

\section{1st Week}

Sideway Jump: Sideway jumps are performed as many as 14 x 5 times.

Squat Jump: Squat Jumps are performed as many as $14 \times 5$ times.

Jumping Different Lengths: Jumps to the different length platforms are applied as many as $14 \mathrm{x} 5$ times.

Increasing Height Jump: Jumps to the platforms with sequenced increasing heights are performed as many as $14 \mathrm{x}$ 5 times.

Decreasing Height Jump: Jumps to the platforms with sequenced decreasing heights are performed as many as 14 $\mathrm{x} 5$ times.

\section{2nd Week}

Sideway Jump: Sideway jumps are performed as many as $16 \times 5$ times.

Squat Jump: Squat Jumps are performed as many as $16 \times 5$ times.

Jumping Different Lengths: Jumps to the different length platforms are applied as many as $16 \mathrm{x} 5$ times.

Increasing Height Jump: Jumps to the platforms with sequenced increasing heights are performed as many as $16 \mathrm{x}$ 5 times.

Decreasing Height Jump: Jumps to the platforms with sequenced decreasing heights are performed as many as 16 $\mathrm{x} 5$ times.

\section{3rd and 4th Weeks}

Sideway Jump: Sideway jumps are performed as many as $18 \times 5$ times.

Squat Jump: Squat Jumps are performed as many as $18 \times 5$ times.

Jumping Different Lengths: Jumps to the different length platforms are applied as many as $18 \mathrm{x} 5$ times.

Increasing Height Jump: Jumps to the platforms with sequenced increasing heights are performed as many as $18 \mathrm{x}$ 5 times.

Decreasing Height Jump: Jumps to the platforms with sequenced decreasing heights are performed as many as 18 $\mathrm{x} 5$ times.

In addition to these exercises, starting from 3rd and 4th weeks the athletes are scheduled to the following exercise:

One foot to left-one foot to right jump: Jumps on left foot for $18 \times 5$ times and jumps on right foot for $18 \times 5$ times are performed.

\section{5th Week}

Sideway Jump: Sideway jumps are performed as many as 20 x 5 times.

Squat Jump: Squat Jumps are performed as many as $20 \times 5$ times. 
Jumping Different Lengths: Jumps to the different length platforms are applied as many as $20 \times 5$ times. 5 times.

Increasing Height Jump: Jumps to the platforms with sequenced increasing heights are performed as many as $20 \mathrm{x}$

Decreasing Height Jump: Jumps to the platforms with sequenced decreasing heights are performed as many as 20 $\mathrm{x} 5$ times.

One foot to left-one foot to right jump: Jumps on left foot for $20 \times 5$ times and jumps on right foot for $20 \times 5$ times are performed.

\section{6th and 7th Weeks}

Sideway Jump: Sideway jumps are performed as many as $22 \times 5$ times.

Squat Jump: Squat Jumps are performed as many as $22 \times 5$ times.

Jumping Different Lengths: Jumps to the different length platforms are applied as many as $22 \times 5$ times.

Increasing Height Jump: Jumps to the platforms with sequenced increasing heights are performed as many as $22 \mathrm{x}$ 5 times.

Decreasing Height Jump: Jumps to the platforms with sequenced decreasing heights are performed as many as 22 $\mathrm{x} 5$ times.

One foot to left-one foot to right jump: Jumps on left foot for $22 \times 5$ times and jumps on right foot for $22 \times 5$ times are performed.

In addition to these exercises, starting from 6th and 7th weeks the athletes are scheduled to the following exercise:

Increasing Height + Squat Jump: Jumps to the platforms with sequenced increasing heights are performed as many as $22 \times 5$ times and for every jump, right after the athlete touches the ground, one squat jump is performed.

\section{8th Week}

Sideway Jump: Sideway jumps are performed as many as $24 \times 5$ times.

Squat Jump: Squat Jumps are performed as many as $24 \times 5$ times.

Jumping Different Lengths: Jumps to the different length platforms are applied as many as $24 \mathrm{x} 5$ times.

Increasing Height Jump: Jumps to the platforms with sequenced increasing heights are performed as many as $24 \mathrm{x}$ 5 times.

Decreasing Height Jump: Jumps to the platforms with sequenced decreasing heights are performed as many as 24 $\mathrm{x} 5$ times.

One foot to left-one foot to right jump: Jumps on left foot for $24 \times 5$ times and jumps on right foot for $24 \times 5$ times are performed.

Increasing Height + Squat Jump: Jumps to the platforms with sequenced increasing heights are performed as many as $24 \times 5$ times and for every jump, right after the athlete touches the ground, one squat jump is performed.

Exercise program of 8 weeks for the experimental group is shown in table 1.

Height measurements of participants are taken with measurement machine of brand Detecto, with 0.1 millimeters of sensitivity. Weight measurements are taken with measurement machine of again brand Detecto with 100 grams of sensitivity. Height and weight measurements are used to calculate body mass indices. Right and left grip force measurements are taken by using Takei brand hand dynamometer. For vertical jumps, Takei brand jump mat and for horizontal jumps, standard meters with $0,1 \mathrm{~cm}$ sensitivity are used. For $30 \mathrm{~m}$ running test measurements, Sinar Photocell Telemetric Stop Watch is used. Additionally, the best of 2 trials of $25 \mathrm{~m}$ and $50 \mathrm{~m}$ free style swimming ranks are recorded by using Colorado Time Systems-SSF device in seconds and split seconds with scoreboard. For the statistical data analysis, non-parametric tests of Wilcoxon and Mann Whitney U tests are used. 
Table 1. Jump movements, definitions and protocols (Nobre et al., 2017)

\begin{tabular}{|c|c|c|c|c|c|c|}
\hline \multirow{2}{*}{\multicolumn{2}{|c|}{$\begin{array}{l}\text { Jumps } \\
\text { Sideway Jump }\end{array}$}} & \multicolumn{5}{|l|}{ Definitions } \\
\hline & & \multicolumn{5}{|c|}{ Jumping to the ground from a $10 \mathrm{~cm}$ platform with two feet towards left and right. } \\
\hline \multicolumn{2}{|c|}{ Squat Jump } & \multicolumn{5}{|c|}{$\begin{array}{l}\text { Jumping from squat position with the help of arms after touching the ground with two feet from } \\
10 \mathrm{~cm} \text { platform. }\end{array}$} \\
\hline \multirow{3}{*}{\multicolumn{2}{|c|}{$\begin{array}{l}\text { Increasing Height Jump } \\
\text { Decreasing Height Jump } \\
\text { One foot to left-one foot to right } \\
\text { jump }\end{array}$}} & \multicolumn{5}{|c|}{ Jumping from the ground to sequenced platforms of $10,20,30$ and $40 \mathrm{~cm}$. } \\
\hline & & \multicolumn{5}{|c|}{ Jumping from the ground to sequenced platforms of $40,30,20$ and $10 \mathrm{~cm}$. } \\
\hline & & \multicolumn{5}{|c|}{ Jumping using only one foot. } \\
\hline Jumpin & g Different Lengths & \multirow{2}{*}{\multicolumn{5}{|c|}{$\begin{array}{l}\text { Jumping to and from platforms of } 30,20,40,10,30 \mathrm{~cm} \text { without touching the ground. } \\
\text { Jumping to } 10,20,30 \text { and } 40 \mathrm{~cm} \text { platforms and then jumping in squat position after touching the } \\
\text { ground. }\end{array}$}} \\
\hline \multicolumn{2}{|c|}{ Increasing Height + Squat Jump } & & & & & \\
\hline \multirow[b]{2}{*}{ Week } & \multirow{2}{*}{\multicolumn{2}{|c|}{ Exercise Type }} & & & & \\
\hline & & & $\begin{array}{l}\text { Set } \\
\text { Qty }\end{array}$ & $\begin{array}{l}\text { Exerc } \\
\text { Qty }\end{array}$ & $\begin{array}{l}\text { Total } \\
\text { Jump Qty }\end{array}$ & $\begin{array}{l}\text { Rest Btw } \\
\text { Exercises }\end{array}$ \\
\hline 1 & \multicolumn{2}{|c|}{$\begin{array}{l}\text { Sideway jump; squat jump; jumping different lengths, increased and } \\
\text { decreased jumps }\end{array}$} & 5 & 14 & 70 & 2-3 min. \\
\hline 2 & \multicolumn{2}{|c|}{$\begin{array}{l}\text { Sideway jump; squat jump; jumping different lengths, increased and } \\
\text { decreased jumps }\end{array}$} & 5 & 16 & 80 & 2-3 min. \\
\hline $3-4$ & \multicolumn{2}{|c|}{$\begin{array}{l}\text { Sideway jump; squat jump; jumping different lengths, increased and } \\
\text { decreased jumps + one foot left, one foot right }\end{array}$} & 5 & 18 & 90 & 2-3 min. \\
\hline 5 & \multicolumn{2}{|c|}{$\begin{array}{l}\text { Sideway jump; squat jump; jumping different lengths, increased and } \\
\text { decreased jumps + one foot left, one foot right }\end{array}$} & 5 & 20 & 100 & 2-3 min. \\
\hline $6-7$ & \multicolumn{2}{|c|}{$\begin{array}{l}\text { Sideway jump; squat jump; jumping different lengths, increased and } \\
\text { decreased jumps + one foot left, one foot right }+ \text { squat jump }\end{array}$} & 5 & 22 & 110 & 2-3 min. \\
\hline 8 & \multicolumn{2}{|c|}{$\begin{array}{l}\text { Sideway jump; squat jump; jumping different lengths, increased and } \\
\text { decreased jumps + one foot left, one foot right }\end{array}$} & 5 & 24 & 120 & 2-3 $\mathrm{min}$. \\
\hline
\end{tabular}

\section{Findings}

Experimental group mean age is $10,33 \pm, 888$ and control group mean age is $10,33 \pm, 985$.

Table 3. Control and experimental group pretest and posttest comparison

\begin{tabular}{|c|c|c|c|c|c|c|c|}
\hline \multirow{2}{*}{ Variables } & \multirow{2}{*}{ Tests } & \multicolumn{3}{|l|}{ Control Group } & \multicolumn{3}{|c|}{ Experimental Group } \\
\hline & & $\mathrm{X} \pm \mathrm{Ss}$ & $\mathbf{Z}$ & $\mathbf{P}$ & $\mathrm{X} \pm \mathrm{Ss}$ & $\mathbf{Z}$ & $\mathbf{P}$ \\
\hline \multirow{2}{*}{ Weight } & Pre Test & $36,18 \pm 5,48$ & \multirow{2}{*}{$-3,065$} & \multirow{2}{*}{, $002 * *$} & $37,64 \pm 7,21$ & \multirow{2}{*}{$-1,336$} & \multirow{2}{*}{, 182} \\
\hline & Post Test & $37,14 \pm 5,65$ & & & $38,50 \pm 7,48$ & & \\
\hline \multirow{2}{*}{ Boy } & Pre Test & $144,17 \pm 4,10$ & \multirow{2}{*}{$-3,035$} & \multirow{2}{*}{, $002 * *$} & $144,33 \pm 6,45$ & \multirow{2}{*}{$-2,136$} & \multirow{2}{*}{, $033 *$} \\
\hline & Post Test & $145,42 \pm 4,14$ & & & $145,25 \pm 8,48$ & & \\
\hline \multirow{2}{*}{ BMI } & Pre Test & $17,39 \pm 2,34$ & \multirow{2}{*}{$-1,961$} & \multirow{2}{*}{, $050 *$} & $17,95 \pm 2,47$ & \multirow{2}{*}{,- 471} & \multirow{2}{*}{,638 } \\
\hline & Post Test & $17,54 \pm 2,36$ & & & $18,13 \pm 2,32$ & & \\
\hline \multirow{2}{*}{ Horizontal jump } & Pre Test & $155,92 \pm 16,03$ & \multirow{2}{*}{$-1,945$} & \multirow{2}{*}{, 052} & $156,42 \pm 15,91$ & \multirow{2}{*}{$-2,982$} & \multirow{2}{*}{, $003 * *$} \\
\hline & Post Test & $157,08 \pm 15,61$ & & & $164,00 \pm 11,48$ & & \\
\hline \multirow{2}{*}{$30 \mathrm{~m}$ sprint } & Pre Test & $5,81 \pm, 421$ & \multirow{2}{*}{,- 985} & \multirow{2}{*}{, 325} & $5,61 \pm, 400$ & \multirow{2}{*}{$-2,120$} & \multirow{2}{*}{, $034 *$} \\
\hline & Post Test & $5,79 \pm, 423$ & & & $5,54 \pm, 502$ & & \\
\hline \multirow{2}{*}{ Vertical jump } & Pre Test & $42,75 \pm 6,90$ & \multirow{2}{*}{$-1,941$} & \multirow{2}{*}{, 052} & $42,92 \pm 8,24$ & \multirow{2}{*}{$-3,070$} & $002 * *$ \\
\hline & Post Test & $43,17 \pm 6,79$ & & & $51,75 \pm 8,18$ & &, 002 \\
\hline & Pre Test & $521,84 \pm 97,15$ & & & $545,17 \pm 122,60$ & -2824 & \\
\hline Anae & Post Test & $537,57 \pm 93,95$ & $-2,140$ & ,006 & $612,32 \pm 130,78$ & 24 & , \\
\hline ce right & Pre Test & $21,72 \pm 4,95$ & & & $22,25 \pm 4,96$ & 59 & \\
\hline Hi & Post Test & $22,40 \pm 4,59$ & & & $26,44 \pm 5,34$ & 9 & \\
\hline orin force left & Pre Test & $22,25 \pm 4,54$ & 66 & $002 * *$ & $23,20 \pm 5,46$ & -3063 & $002 * *$ \\
\hline Hand grip Iorce lent & Post Test & $22,70 \pm 4,66$ & 00 &, $002 \cdots$ & $26,75 \pm 5,30$ & $-5,003$ &, $002 \cdots$ \\
\hline & Pre Test & $18,37 \pm 2,49$ & & & $17,80 \pm 2,00$ & & $015 *$ \\
\hline $25 \mathrm{~m}$ tree style & Post Test & $18,30 \pm 3,20$ & $-1,698$ & ,090 & $16,57 \pm 2,17$ & $-2,432$ &, $015^{*}$ \\
\hline $50 \mathrm{~m}$ free style & Pre Test & $38,56 \pm 4,72$ & $-1,571$ & .116 & $37,19 \pm 4,00$ & $-3,059$ &, $002 * *$ \\
\hline & Post Test & $38,46 \pm 4,76$ & $-1,5 / 1$ &, 110 & $36,08 \pm 4,19$ & $-5,059$ & \\
\hline
\end{tabular}

$(* p<0,05 ; * * p<0,01)$

As can be seen in Table 3, as per in the pretest and posttest comparisons of control group, except for horizontal jump, 30 $\mathrm{m}$ sprint, vertical jump, $25 \mathrm{~m}$ and $50 \mathrm{~m}$ free style ranks, other parameters showed statistically significant differences $(\mathrm{p}<0,05)$. As for experimental group, except for body weight and body mass index (BMI), other parameters showed statistically significant differences $(\mathrm{p}<0,05)$. 
Table 4. Comparison of pretest and posttest results of control and experimental groups

\begin{tabular}{|c|c|c|c|c|c|}
\hline Variables & Tests & $\begin{array}{l}\text { Control Group } \\
\mathrm{X} \pm \mathrm{Ss}\end{array}$ & $\begin{array}{l}\text { Experimental Group } \\
\text { X } \pm \text { Ss }\end{array}$ & $\mathbf{Z}$ & $\mathbf{P}$ \\
\hline \multirow{2}{*}{ Weight } & Pre Test & $36,18 \pm 5,48$ & $37,64 \pm 7,21$ &,- 376 & ,707 \\
\hline & Post Test & $37,14 \pm 5,65$ & $38,50 \pm 7,48$ &,- 347 & ,729 \\
\hline \multirow{2}{*}{ Boy } & Pre Test & $144,17 \pm 4,10$ & $144,33 \pm 6,45$ &,- 377 & ,706 \\
\hline & Post Test & $145,42 \pm 4,14$ & $145,25 \pm 8,48$ &,- 781 &, 435 \\
\hline \multirow{2}{*}{ BMI } & Pre Test & $17,39 \pm 2,34$ & $17,95 \pm 2,47$ &,- 404 & 686 \\
\hline & Post Test & $17,54 \pm 2,36$ & $18,13 \pm 2,32$ &,- 866 & ,386 \\
\hline \multirow{2}{*}{ Horizontal jump } & Pre Test & $155,92 \pm 16,03$ & $156,42 \pm 15,91$ &,- 087 & ,931 \\
\hline & Post Test & $157,08 \pm 15,61$ & $164,00 \pm 11,48$ & $-1,069$ & ,285 \\
\hline \multirow{2}{*}{$30 \mathrm{~m}$ sprint } & Pre Test & $5,81 \pm, 421$ & $5,61 \pm, 400$ & $-1,097$ & 273 \\
\hline & Post Test & $5,79 \pm, 423$ & $5,54 \pm, 502$ & $-1,848$ &, 065 \\
\hline \multirow{2}{*}{ Vertical jump } & Pre Test & $42,75 \pm 6,90$ & $42,92 \pm 8,24$ &,- 462 & 644 \\
\hline & Post Test & $43,17 \pm 6,79$ & $51,75 \pm 8,18$ & $-2,545$ &, $011 *$ \\
\hline \multirow{2}{*}{ Anaerobic power } & Pre Test & $521,84 \pm 97,15$ & $545,17 \pm 122,60$ &,- 693 & ,488 \\
\hline & Post Test & $537,57 \pm 93,95$ & $612,32 \pm 130,78$ & $-1,501$ & ,133 \\
\hline \multirow{2}{*}{ Hand grip force right } & Pre Test & $21,72 \pm 4,95$ & $22,25 \pm 4,96$ &,- 346 & ,729 \\
\hline & Post Test & $22,40 \pm 4,59$ & $26,44 \pm 5,34$ & $-1,733$ & ,083 \\
\hline \multirow{2}{*}{ Hand grip force left } & Pre Test & $22,25 \pm 4,54$ & $23,20 \pm 5,46$ &,- 549 &, 583 \\
\hline & Pre Test & $22,70 \pm 4,66$ & $26,75 \pm 5,30$ & $-1,935$ &, 053 \\
\hline \multirow{2}{*}{$25 \mathrm{~m}$ free style } & Pre Test & $18,37 \pm 2,49$ & $17,80 \pm 2,00$ &,- 520 & 603 \\
\hline & Post Test & $18,30 \pm 3,20$ & $16,57 \pm 2,17$ & $-1,559$ &, 119 \\
\hline \multirow{2}{*}{$50 \mathrm{~m}$ free style } & Pre Test & $38,56 \pm 4,72$ & $37,19 \pm 4,00$ &,- 751 & ,453 \\
\hline & Pre Test & $38,46 \pm 4,76$ & $36,08 \pm 4,19$ & $-1,617$ &, 106 \\
\hline
\end{tabular}

$(\mathrm{p}<0,05)$

As can be seen in Table 4, in the pretest and posttest result comparison, except for vertical jump parameter, other parameters showed no statistically significant difference in favor of the experimental group $(\mathrm{p}>0,05)$.

\section{Conclusion}

Even though there is no statistical significance between the group of swimmers of age 10-11 who underwent 8-week plyometric exercise and the control group as per pretest and posttests, there is indeed difference. When literature is reviewed, in developing jumping skills, the power exercises which the athletes of this age do with their own body weights stand out (Bompa, 2013; Kish et al., 2015). Additionally, it is underscored that there is also an effect on the physiological and physical attributes of the athletes (Akcapinar, 2014; Oztin et al., 2003). Some researchers suggest low intensity jump trainings to age group 12-14 and mid intensity jump training to ages above 14 in order to form a basis for their future power trainings (Mentes et al., 1989). There are also studies in the literature showing plyometric exercises performed during younger ages (Nobre et al., 2017). The fact that plyometric exercises especially contributed to the vertical jump parameter in this study supports what is seen in the literature. The literature frequently displays the physical and physiological effects of plyometric exercises however studies of performance are limited and showing contradictory results. In the study in which Gul et al. (2017) checked the effect of 8 weeks of plyometric exercises on service hit rates in tennis, it is expressed to have no effect. In the study of Olcucu (2012) it is found that plyometric exercises do have an effect on service hit rates. While the performed studies vary in results as per the branch of sports, it is observed that the limited amount of studies performed on swimming shows that plyometric exercises have a small amount of effect. Wilson (2004) did not find the effect of plyometric exercises of swimmers on loop turns ( $\mathrm{p}>0,05$ ). Potdevin et al. (2011) put forward in their study that 6 weeks of plyometric exercises have an effect on start performance and loop turns of swimmers. Bishop et al. (2009) reviewed the effects of 8 weeks of plyometric exercise on the start performance of swimmers and observed that it indeed has an effect. In our study, even though it is not statistically significant, the decrease in the duration of $25 \mathrm{~m}$ and $50 \mathrm{~m}$ free style swims showed that plyometric exercises do have an effect on the performance even though slightly. In the light of all these studies, considering that even split seconds are of vital importance for world records, it can be reckoned that regularly performed plyometric exercises have a positive effect on the performance of athletes due to their effect on athlete's explosive power and maximal power. Another reason for this is that plyometric exercises also affect starts and turns and foot hits in the water, and as a result it offers improvements in duration, even though slightly. For all aforementioned reasons the study is also aims to enlighten trainers of swimmers. 


\section{References}

Akcapinar, F. (2014). The Effect Of Plyometric Training On Balance And Soccer-Specific Of Aged 11-12 Children, Inonu University Health Sciences Institute, Doctorate Thesis

Bishop, D. C., Smith, R. J., Smith, M. F., \& Rigby, H. E. (2009). Effect of plyometric training on swimming block start performance in adolescents. The Journal of Strength \& Conditioning Research, 23(7), 2137-2143. https://doi.org/10.1519/JSC.0b013e3181b866d0

Bompa, T. O. (2013). Quick Force Training in sports (Plyometric for Upper Level Force Development), (Translated by: Eda Tuzuman), Sports Publisher and Bookstore, Ankara

Bozdogan, A. (2003). Physiology, mechanics and method of swimming, Ilpress Printing and Publishing, Istanbul

Bozdogan, A., \& Ozuak, A. (2003). Basic swimming with the styles. Ilpress Printing and Publishing, Istanbul

Donald, A., \& Myer, G. D. (2013). Plyometrics, Human Kinetics.

Gul, G. K., Konyali, M., \& Gul, M. (2017). The Effect Of Plyometric Training On The Tennis Service Hits, Journal of Physical Education and Sports Studies, 9(1), 67-73.

Kish, K., Mezil, Y., Ward, W. E., Klentrou, P., \& Falk, B. (2015). Effects of plyometric exercise session on markers of bone turnover in boys and young men. European journal of applied physiology, 115(10), 2115-2124. https://doi.org/10.1007/s00421-015-3191-z

Marinho, D. A., Amorim, R. A., Costa, A. M., Marques, M. C., Pérez, T. J. A., \& Neiva, H. P. (2011). "Anaerobic” critical velocity and swimming performance in young swimmers.

Mentes, Ç., Turgut, M., Hasçelik, Z., \& Özker, R. (1989). Pliometric, Power Acceptable Form, Turkish Journal of Sports Medicine, 24(2), 55-62.

Morpa Sports Encyclopedia (2003). Vol. 3, Istanbul.

Nobre, G. G., de Almeida, M. B., Nobre, I. G., dos Santos, F. K., Brinco, R. A., Arruda-Lima, T. R., ... Moura-dos-Santos, M. A. (2017). Twelve Weeks of Plyometric Training Improves Motor Performance of 7-to 9-Year-Old Boys Who Were Overweight/Obese: A Randomized Controlled Intervention. The Journal of Strength \& Conditioning Research, 31(8), 2091-2099. https://doi.org/10.1519/JSC.0000000000001684

Olcucu, B. (2012). Investigation of the effect of plyometric trainings on arm and leg strenght, the strokes speed of service, forehand and bachand and the percentages of shot accuracy, Marmara University Health Sciences Institute, Doctorate Thesis

Oztin, S., Erol, A. E., \& Pulur, A. (2003). The Effect Of Pliometric And Explosive Power Trainings On The Physical And Physiological Characteristics Of 15-16 Ages Group Basketball Players, Gazi Journal of Physical Education and Sport Sciences (Gazi BESBD), VIII(1)1, 41-52.

Potdevin, F. J., Alberty, M. E., Chevutschi, A., Pelayo, P., \& Sidney, M. C. (2011). Effects of a 6-week plyometric training program on performances in pubescent swimmers. The Journal of Strength \& Conditioning Research, 25(1), 80-86. https://doi.org/10.1519/JSC.0b013e3181fef720

Sevim, Y. (1991). Condition Training, 1st Edition, Gazi Office and Bookstore Publications, Ankara, 35-63.

Troup, J. P. (1999). The physiology and biomechanics of competitive swimming. Clinics in Sport Medicine, 18(2).

Tuzen, B., Muniroglu, S., \& Tanilkan, K. (2005). The Investigation Of Short Distance Swimmer To Compare Between 50 Meter Craftstyle Swimming And 30 Meter Sprint Test Results, Spormetre Beden Eğitimi ve Spor Bilimleri Dergisi, (3), 97-99.

Wilson, M., Adams, K. J., \& Stamford, B. A. (2004). Aquatic plyometrics and the freestyle flip turn. Medicine \& Science in Sports \& Exercise, 36(5), 206.

Yapici, A., \& Cengiz, C. (2015). The Relationship Between Lower Extremity Wingate Anaerobic Test (WAnT) and 50m Freestyle Swimming Performance. International Journal of Science Culture and Sport (IntJSCS), 3(3), $44-54$. https://doi.org/10.14486/IJSCS257

\section{Copyrights}

Copyright for this article is retained by the author(s), with first publication rights granted to the journal.

This is an open-access article distributed under the terms and conditions of the Creative Commons Attribution license which permits unrestricted use, distribution, and reproduction in any medium, provided the original work is properly cited. 